\title{
MAZUR'S THEOREM ON SEQUENTIALLY CONTINUOUS LINEAR FUNCTIONALS
}

\author{
J. R. ISBELL AND E. S. THOMAS, JR.
}

1. Introduction. A linear topological space $A$ satisfies Mazur's theorem provided every sequentially continuous linear functional on $A$ is continuous. There have been a number of investigations of conditions on a space $A$ of real valued functions on a set $X$ in order that $A$, with the topology of pointwise convergence on $X$, should satisfy Mazur's theorem. S. Mazur opened the question for spaces $A=C(X)$ and gave a strong theorem [3] whose statement is a little too complicated to give here. The fact that Mazur's theorem always holds for $C(X)$ if $X$ is compact seems first to have been proved by V. Pták in 1956 (see [2]); the first publication seems to be in [4, Theorem C and footnote 8].

A linear space of functions $A$ on a set $X$ determines a uniform structure on $X$. Relative to this structure $X$ has a completion, $\hat{X}$. As is well known, a linear functional on $A$ which is continuous relative to the weak topology is representable in $X$, i.e., a finite linear combination of evaluations at points of $X$.

In this paper we split the problem of Mazur's theorem into two parts as follows. (1) For which spaces $A$ is every sequentially continuous linear functional on $A$ representable in $\hat{X}$ ? (2) Which points $p$ of $\hat{X}$ yield sequentially continuous linear functionals? We give three theorems, all assuming that $A$ is closed under certain operations. The weakest assumptions are, alternatively, that $A$ is closed under the lattice operations $f \vee g, f \wedge g$, and the bounding operations $(f \wedge n) \vee-n$, or that $A$ is closed under composition with real entire functions vanishing at 0 ; either assumption takes care of problem (1). For (2) we assume also that $A$ contains the constant functions; then the points $p$ in question are those for which every countable set of functions in $A$ vanishing at $p$ has a common zero in $X$. Finally, replacing the entire functions with $C^{\infty}$ functions, we show that if every function locally belonging to $A$ belongs to $A$ then evaluation at any point of $\hat{X}$ is sequentially continuous.

We remark that examples in [2] show that some linear subspaces of $C(X)$ may admit sequentially continuous linear functionals which are not representable in $\hat{X}$; also, that the two papers by Isbell and Thomas, respectively, which are cited in [2], are combined in this paper.

Received by the editors May 5, 1962. 
2. Theorems. We say $A$ is lattice-closed if $A$ is closed under the two unary operations $|f|,(f \wedge 1) \vee-1$; since we are also assuming $A$ is a linear space, it is closed under $f \vee g, f \wedge g$, and $(f \wedge n) \vee-n$ as well. We say $A$ is analytically closed if it is closed under all the unary operations $h \circ f$, where $h$ is an entire function real on the real line and 0 at $0 . A$ is locally determined if every function defined on $X$ which agrees with some function in $A$ on a neighborhood of each point belongs to $A$. We assume for convenience that $A$ separates points on $X$.

The entire functions to be used below are as follows: Functions $\alpha_{n}$ within $1 / n$ of $|x|$ on $[-n, n]$ (these may be polynomials); functions $\beta_{n}$ within $1 / n$ of $(x \wedge n) \vee-n$ on the whole line; the square function $g(x)=x^{2}$; a function $h$ with $h(0)=h(+\infty)=0 \neq h(-1)$. The existence of the $\beta_{n}$ follows from Carleman's approximation theorem [1].

We use a special case of a lemma of H. H. Corson (see [2]); if $A$ and $B$ are linear spaces of functions on a set $X$, and $A$ is a uniformly dense subset of $B$, then every sequentially continuous linear functional on $A$ in the weak topology has a sequentially continuous extension over $B$.

Theorem 1. If $A$ is (i) lattice-closed or (ii) analytically closed, then every sequentially continuous linear functional on $A$ is representable in $\hat{X}$.

Proof. Let $A^{*}$ be the subspace of $A$ consisting of all the bounded functions. $A^{*}$ determines a uniform structure on $X$ and a compact completion $\bar{X}$; so we may regard $A^{*}$ as contained in $C(\bar{X})$. Either hypothesis implies (using the functions $\alpha_{n}$ ) that the closure of $A^{*}$ in the norm topology is a lattice. Therefore it is either $C(\bar{X})$ or a closed hyperplane consisting of all functions vanishing at a point.

Then for any sequentially continuous functional $\phi$ on $A$, the restriction $\phi \mid A^{*}$ has at least one sequentially continuous extension over $C(\bar{X})$ in the topology of pointwise convergence on $X$. Since the topology of pointwise convergence on $\bar{X}$ is finer, it follows from Pták's theorem $[2 ; 4]$ that $\phi \mid A^{*}$ is representable in $\bar{X}$. To conclude, it suffices to show that if $\psi$ is a sequentially continuous linear functional on $A$ and $p$ is a point of $\bar{X}$ such that $\psi(f)=f(p)$ for all $f$ in $A^{*}$, then there is a net of points $x_{\lambda}$ of $X$ such that $f\left(x_{\lambda}\right)$ converges to $\psi(f)$ for all $f$ in $A$. (That is, the "good" points of $\bar{X}$ are points of $\hat{X}$.) For this we take any net $\left\{x_{\lambda}\right\}$ converging to $p$ in $\bar{X}$; and we consider the functions $f_{n}$ defined (case (i)) as $(f \wedge n) \vee-n$, or (case (ii)) as $\beta_{n} \circ f$. For any positive $\epsilon$, choose an index $m>\epsilon^{-1}$ such that $m-1 / m>|\psi(f)|$ $+\epsilon$, and $\left|\psi\left(f_{m}\right)-\psi(f)\right|<\epsilon$. The numbers $f_{m}\left(x_{\lambda}\right)$ converge to $\psi\left(f_{m}\right)$; 
so they are finally within $\epsilon$ of $\psi(f)$. In particular $\left|f_{m}\left(x_{\lambda}\right)\right|<m-1 / m$, finally in $\lambda$. Hence for these $\lambda,\left|f_{m}\left(x_{\lambda}\right)-f\left(x_{\lambda}\right)\right|<1 / m$. Thus $\left|f\left(x_{\lambda}\right)-\psi(f)\right|<\epsilon+1 / m<2 \epsilon$. Since $\epsilon$ is arbitrary, this proves $f\left(x_{\lambda}\right) \rightarrow$ $\psi(f)$, as was to be shown.

THEOREM 2. Let $A$ be as in Theorem 1 and contain the constants, and let $p$ be a point of $\hat{X}$. Then evaluation at $p$ is sequentially continuous on $A$ if and only if every sequence in $A$ vanishing identically at $p$ vanishes identically at some point of $X$.

Proof. Assume (ii) that $A$ is analytically closed, and suppose $\left\{f_{n}\right\}$ is a sequence vanishing at $p$ but, for each $x$ in $X$, some $f_{n}(x) \neq 0$. Then defining $g_{n}$ as $n \sum_{i=1}^{i=n} f_{i}^{2}$, we have $g_{n}(p)=0$ but $g_{n} \rightarrow+\infty$ on $X$. If $A$ contains the constants, define $h_{n}=h \circ\left(g_{n}-1\right)$, where $h(0)$ $=h(+\infty)=0, h(-1) \neq 0$. Then $h_{n} \rightarrow 0$ on $X$ but $h_{n}(p)=h(-1)$; so evaluation at $p$ is not sequentially continuous.

Conversely, if evaluation at $p$ is not sequentially continuous, we may pick a sequence of non-negative functions $f_{n}$ converging to 0 on $X$ but with $f_{n}(p)=1$ for all $n$. Setting $j_{n}(x)=1-f_{n}(x)$, we have a sequence all vanishing at $p$ but not all vanishing at any point of $X$

For case (i), we simply use $|x|$ instead of $x^{2}$ and $x \wedge 0$ instead of $h$.

Theorem 3. If $A$ is locally determined and (i) lattice-closed or (ii) closed under composition with real $C^{\infty}$ functions vanishing at 0 , then evaluation at each point of $\hat{X}$ is sequentially continuous.

Proof. We show first that the constants can be adjoined to $A$, and the hypotheses preserved, without changing $\hat{X}$; in fact we need only take the functions $f+k, f$ in $A, k$ constant. Since $A$ separates points on $X$, there is at most one point $x_{0}$ at which all functions in $A$ vanish. Suppose $g$ is a function on $X$ which locally has the form $f_{\alpha}+k_{\alpha}$, for various $f_{\alpha}$ in $A$ and various constants $k_{\alpha}$. If $g=f_{0}+k_{0}$ near $x_{0}$, then $g-k_{0}$ agrees with a function in $A$ near $x_{0}$, and also everywhere else; for at any other point $x$ of $X$ there is a function in $A$ taking a nonzero value and, by (i) or (ii), a function in $A$ taking a nonzero constant value near $x$. Thus the space of all $f+k$ is locally determined. Entirely similar arguments show that it retains property (i) or (ii). So we may assume $A$ already contains the constants.

Suppose for some $p$ in $\hat{X}$ that evaluation at $p$ is not sequentially continuous. As in the proof of Theorem 2, there is a sequence of nonnegative functions $f_{n}$ in $A$, each vanishing at $p$, with $f_{n}(x)$ forming an unbounded increasing sequence for each $x$ in $X$. Let $g$ be a nonnegative, real, $C^{\infty}$ function vanishing on $(-\infty, 1 / 2]$ and identically 1 on $[1, \infty)$, and consider any $x$ in $X$. Near $x$ almost all $f_{n} \geqq 1$, hence 
the equation $f(x)=\sum\left(1-g \circ f_{n}(x)\right)$ defines a function which, near any $x$ in $X$, is a finite sum of functions in $A$ and therefore is in $A$.

On the other hand $f$ cannot be extended over $p$, for near $p$ arbitrarily many $g \circ f_{n}$ are zero and $f$ is arbitrarily large. The contradiction establishes the theorem.

\section{REFERENCES}

1. T. Carleman, Sur un théorème de Weierstrass, Ark. Mat. 20 (1927), 5 pp.

2. J. Isbell, Mazur's theorem, Proceedings of the Topological Symposium in Commemoration of E. Cech, Prague, 1962, pp. 221-225.

3. S. Mazur, Sur la structure des fonctionnelles linéaires dans certains espaces $(L)$, Ann. Soc. Polon. Math. 19 (1946), 241.

4. S. Mrowka, On the form of pointwise continuous positive functionals and isomorphisms of function spaces, Studia Math. 21 (1961), 1-14.

UNIVERSITY OF WASHINGTON AND

UNIVERSITY OF CALIFORNIA, RIVERSIDE 